\title{
Functional bowel disorders and functional abdominal pain
}

\author{
W G Thompson, G F Longstreth, D A Drossman, K W Heaton, E J Irvine,
} S A Müller-Lissner

Chair, Committee on Functional Bowel Disorders and Functional Abdominal Pain, Multinational Working Teams to Develop Diagnostic Criteria for Functional Gastrointestinal Disorders (Rome II), Emeritus Professor of Medicine,

University of Ottawa, Canada

W G Thompson

Co-Chair, Committee on Functional Bowel

Disorders and

Functional Abdominal

Pain, Multinational

Working Teams to

Develop Diagnostic

Criteria for Functional

Gastrointestinal

Disorders (Rome II),

Kaiser Permanente,

San Diego, CA, USA

G F Longstreth

Division of Digestive Diseases,

University of North

Carolina, Chapel Hill,

NC, USA

D A Drossman

Department of

Medicine,

University of Bristol,

Bristol, UK

K W Heaton

Department of

Medicine,

McMaster University,

Ontario, Canada

E J Irvine

Department of

Medicine,

Park-Klinik,

Weissensee, Berlin,

Germany

S A Müller-Lissner

Correspondence to:

W Grant Thompson, MD,

7 Nesbitt Street, Nepean,

Ontario K2H 8C4, Canada.

\section{Abstract}

The Rome diagnostic criteria for the functional bowel disorders and functional abdominal pain are used widely in research and practice. A committee consensus approach, including criticism from multinational expert reviewers, was used to revise the diagnostic criteria and update diagnosis and treatment recommendations, based on research results. The terminology was clarified and the diagnostic criteria and management recommendations were revised. A functional bowel disorder (FBD) is diagnosed by characteristic symptoms for at least 12 weeks during the preceding 12 months in the absence of a structural or biochemical explanation. The irritable bowel syndrome, functional abdominal bloating, functional constipation, and functional diarrhea are distinguished by symptombased diagnostic criteria. Unspecified FBD lacks criteria for the other FBDs. Diagnostic testing is individualized, depending on patient age, primary symptom characteristics, and other clinical and laboratory features. Functional abdominal pain (FAP) is defined as either the FAP syndrome, which requires at least six months of pain with poor relation to gut function and loss of daily activities, or unspecified FAP, which lacks criteria for the FAP syndrome. An organic cause for the pain must be excluded, but aspects of the patient's pain behavior are of primary importance. Treatment of the FBDs relies upon confident diagnosis, explanation, and reassurance. Diet alteration, drug treatment, and psychotherapy may be beneficial, depending on the symptoms and psychological features.

(Gut 1999;45(Suppl II):II43-II47)

Keywords: functional bowel disorder; functional constipation; functional diarrhea; irritable bowel syndrome; functional abdominal pain; functional abdominal bloating; Rome II

The functional bowel disorders and functional abdominal pain are common and cause much suffering. As these entities are identified by symptoms and patient care is highly individualized, a symptom-based classification has great importance, particularly for use in clinical trials (table 1).

The 1998 Working Team assessed the terminology and results of relevant clinical research in order to revise the diagnostic criteria,
Table 1 Functional gastrointestinal disorders

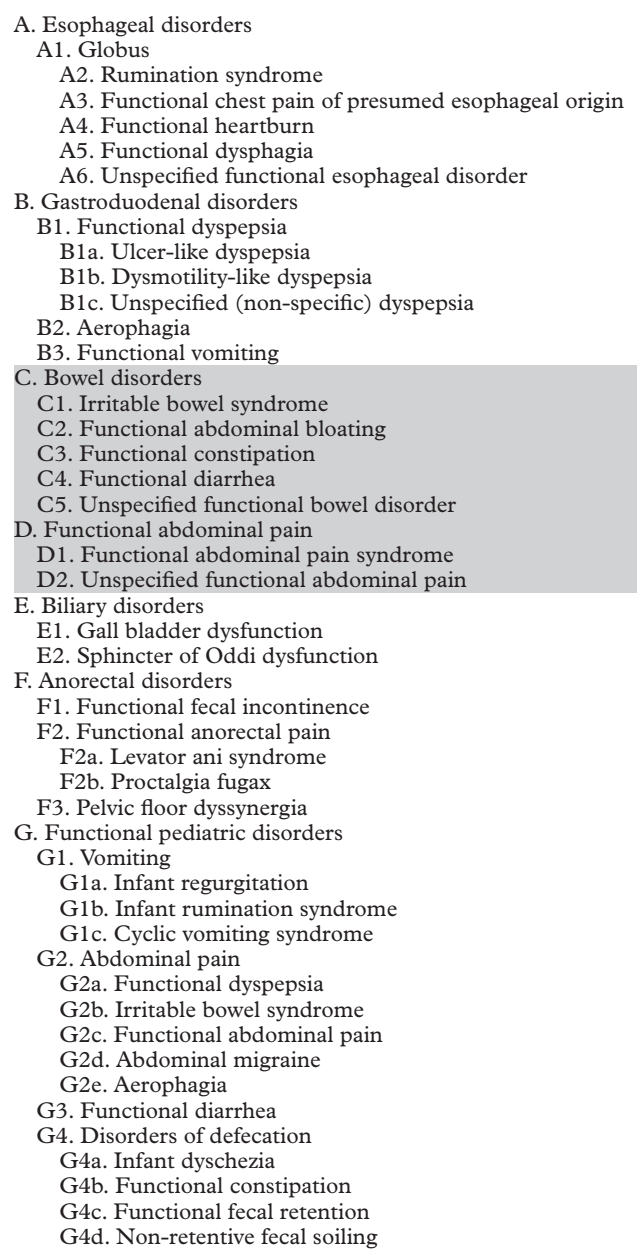

comment further on diagnosis, and summarize treatment recommendations.

\section{Functional bowel disorders}

A functional bowel disorder (FBD) is a functional gastrointestinal disorder with symptoms attributable to the mid or lower gastrointestinal tract, including the irritable bowel syndrome (IBS), functional abdominal bloating, functional constipation, functional diarrhea, and unspecified functional bowel disorder.

Subjects with a FBD may be divided into the following groups:

Abbreviations used in this paper: $\mathrm{FBD}$, functional bowel disorder; IBS, irritable bowel syndrome; FAPS, functional abdominal pain syndrome. 
(1) non-patients: those who have never sought health care for the FBD;

(2) patients: those who have sought care for the FBD; (a) incident cases: those who have sought care for the FBD for the first time in the past year; $(b)$ prevalent cases: those who have ever sought care for the FBD.

Symptoms of a FBD must have been present for 12 weeks or more within the past 12 months; the 12 weeks need not be consecutive. The diagnosis always presumes the absence of a structural or biochemical explanation for the symptoms.

The Working Team changed the definitions of IBS, functional abdominal bloating and functional constipation from a "disorder" to "disorders" to acknowledge multiple pathophysiologic possibilities. For IBS, "discomfort" was added to "pain" to broaden symptom description, and "distension" was deleted.

To clarify how discomfort and pain are temporally related to a change in frequency and form of stool, "onset" was added to the relevant symptom features. The symptom criteria for IBS were changed (a) by designating the nonpain-related, the second part of the previous criteria, as nonessential due to their poor clustering in factor analyses ${ }^{12}$; (b) their lesser prevalence in men $^{3}$; and $(c)$ the partial duplication in the retained, pain-related criteria. Furthermore, these symptoms were clarified by replacing the term "altered" with "abnormal." The criteria for functional constipation were expanded due to the overlap between it and the functional anorectal disorders (see Functional disorders of the anus and rectum).

\section{C1. Irritable bowel syndrome}

IBS comprises a group of functional bowel disorders in which abdominal discomfort or pain is associated with defecation or a change in bowel habit, and with features of disordered defecation.

Surveys of Western populations have revealed IBS in $15-20 \%$ of adolescents and adults, with a higher prevalence in women; the prevalence is variable in other populations. ${ }^{4}$ IBS has a chronic relapsing course and overlaps with other functional gastrointestinal disorders. ${ }^{5}$ It accounts for high direct medical expenses ${ }^{6}$ and indirect costs, including absenteeism from work. ${ }^{5}$

DIAGNOSTIC CRITERIA

At least 12 weeks, which need not be consecutive, in the preceding 12 months of abdominal discomfort or pain that has two of three features:

(1) Relieved with defecation; and/or

(2) Onset associated with a change in frequency of stool; and/or

(3) Onset associated with a change in form (appearance) of stool.

The following symptoms cumulatively support the diagnosis of IBS:

- abnormal stool frequency (for research purposes "abnormal" may be defined as $>3 /$ day and $<3 /$ week);
Table 2 Supportive symptoms of the irritable bowel syndrome

1. Fewer than three bowel movements a week

2. More than three bowel movements a day

3. Hard or lumpy stools

4. Loose (mushy) or watery stools

5. Straining during a bowel movement

6. Urgency (having to rush to have a bowel movement)

7. Feeling of incomplete bowel movement

8. Passing mucus (white material) during a bowel movement 9. Abdominal fullness, bloating or swelling Diarrhea-predominant

or more of 2,4 , or 6 and none of 1,3 , or 5 Constipation-predominant

1 or more of 1,3 , or 5 and none of 2,4 , or 6

- abnormal stool form (lumpy/hard or loose/ watery stool);

- abnormal stool passage (straining, urgency, or feeling of incomplete evacuation);

- passage of mucus;

- bloating or feeling of abdominal distension.

These symptoms can be used to subclassify patients with predominant diarrhea or constipation for entry into clinical trials (table 2).

DIAGNOSTIC COMMENTS

The validity of the symptom criteria is supported by patient studies, ${ }^{378}$ factor analyses on non-patients ${ }^{12}$ and long term patient follow up. ${ }^{9}$ The history may also reveal postprandial symptom exacerbation, ${ }^{10}$ and occasionally, incontinence and nocturnal pain. Symptom worsening during menses and other features can falsely suggest a gynecologic explanation for "chronic pelvic pain." ${ }^{11}$ Other non-gastrointestinal somatic symptoms are common. ${ }^{5}$ Colonic transit time can be estimated by rating self-reported stool appearance according to a stool form scale. ${ }^{12}$ Fever, rectal bleeding, or weight loss require consideration of another disorder. ${ }^{13}$ Abdominal examination reveals no abnormality. Large bowel structural examination is recommended to support a symptom-based diagnosis. The choice of such examinations should be guided by the age and gender of the patient, the nature and duration of symptoms, and other factors. ${ }^{8}{ }^{14}$

\section{TREATMENT RECOMMENDATIONS}

A confident diagnosis, explanation, and reassurance are vital therapeutic tools. A graded, multicomponent approach is advised, depending on the dominant symptoms, their severity and psychosocial factors. ${ }^{4}$ Although rigorous therapeutic trials are scarce, popular therapy includes the use increased dietary fiber such as wheat bran or bulking agents for constipation, loperimide or diphenoxylate for diarrhea, and anticholinergic/antispasmodic agents or lowdose antidepressants for pain. ${ }^{4}$ Psychological or behavioral treatments may help some patients.

\section{C2. Functional abdominal bloating}

Functional abdominal bloating comprises a group of functional bowel disorders which are dominated by a feeling of abdominal fullness or bloating and without sufficient criteria for another functional gastrointestinal disorder.

Few studies adequately separate bloating from IBS and other functional disorders, but it occurs in about $15 \%$ of community-based 
populations, usually with a female predominance. ${ }^{1516}$

DIAGNOSTIC CRITERIA

At least 12 weeks, which need not be consecutive, in the preceding 12 months of:

(1) Feeling of abdominal fullness, bloating, or visible distension; and

(2) Insufficient criteria for a diagnosis of functional dyspepsia, IBS, or other functional disorder.

DIAGNOSTIC COMMENTS

Functional bloating is usually absent on awakening and worsens throughout the day. It may be intermittent and related to ingestion of specific foods. Excessive burping or farting may be present, but these are not necessarily related to the bloating. Diarrhea, weight loss, or nutritional deficiency should alert the physician to investigate for another disorder.

TREATMENT RECOMMENDATIONS

There is no proved effective therapy for functional bloating, and its cause is unknown, so only education and reassurance are recommended. The common practice of restricting certain "gas-forming" foods may be beneficial but even patients with confirmed lactase deficiency can drink $250 \mathrm{ml}$ milk with no or negligible bloating. ${ }^{17}$

\section{C3. Functional constipation}

Functional constipation comprises a group of functional disorders which present as persistent difficult, infrequent or seemingly incomplete defecation.

Constipation occurs in up to $20 \%$ of populations, depending on demographic factors, sampling and the definition used. It is more common in women and is usually found to increase with age. ${ }^{518}$

DIAGNOSTIC CRITERIA

At least 12 weeks, which need not be consecutive, in the preceding 12 months of two or more of:

(1) Straining in $>1 / 4$ defecations;

(2) Lumpy or hard stools in $>1 / 4$ defecations;

(3) Sensation of incomplete evacuation in $>1 / 4$ defecations;

(4) Sensation of anorectal obstruction/ blockade in $>1 / 4$ defecations

(5) Manual maneuvers to facilitate $>1 / 4$ defecations (e.g., digital evacuation, support of the pelvic floor); and/or

(6) $<3$ defecations/week.

Loose stools are not present, and there are insufficient criteria for IBS.

DIAGNOSTIC COMMENTS

The physician should clarify what the patient means by constipation, as patients describe it in various ways. ${ }^{19}$ Many, if not most patients actually have the rectal symptoms of IBS with or without lumpy stools. ${ }^{20}$ Evaluation of the patient's general health, psychological status, use of constipating medications, dietary fiber intake, and medical illnesses (e.g., hypothyroidism) is important. In patients who do not respond to fiber supplementation, measurements of whole gut transit time ${ }^{21}$ and anorectal function ${ }^{22}$ may be indicated to place them in a physiological subgroup.

TREATMENT RECOMMENDATIONS

Dietary fiber increases fecal bulk by providing indigestible matter and promoting fecal water holding and bacterial proliferation. ${ }^{23}$ Other useful bulking agents include psyllium, methylcellulose, and calcium polycarbophil. Severely constipated patients may respond to polyethelene glycol solution. ${ }^{24}$ Otherwise, stimulant laxatives such as bisacodyl, sodium picosulphate, or sennosides may be tried. Specific treatment for patients in the anorectal dysfunction subgroup is discussed in Functional disorders of the anus and rectum.

\section{C4. Functional diarrhea}

Functional diarrhea is continuous or recurrent passage of loose (mushy) or watery stools without abdominal pain.

A British population survey of stool form (a scale previously validated against symptoms and transit time ${ }^{12}$ ) revealed liquid stools were the predominant type described by $5.3 \%$ of men and $4.3 \%$ of women. ${ }^{25}$ Liquid stools were more common in women under 50 years of age than in older women.

DIAGNOSTIC CRITERIA

At least 12 weeks, which need not be consecutive, in the preceding 12 months of:

(1) Liquid (mushy) or watery stools;

(2) Present $>3 / 4$ of the time; and

(3) No abdominal pain.

DIAGNOSTIC COMMENTS

Pseudodiarrhea (frequent defecation and urgency with solid stools) must be distinguished from diarrhea. Chronic diarrhea without pain is caused by many diseases indistinguishable by history, which should be excluded by diagnostic testing. Basic evaluation includes routine blood and stool tests plus sigmoidoscopy with biopsy. Features atypical for a functional disorder (e.g., large volume stools, rectal bleeding, nutritional deficiency, and weight loss) call for more extensive studies of intestinal structure and function-for example, radiography, duodenal biopsy, and serum hormone assay.

TREATMENT RECOMMENDATIONS

Discussion of possible psychosocial factors, symptom explanation, and reassurance are important. Restriction of foods which seem provocative may help. Empiric antidiarrheal therapy (e.g., diphenoxylate or loperimide) is usually effective, especially if taken prophylactically, such as before meals. The occasional patient responds to cholestyramine. Fortunately, spontaneous remissions are common. ${ }^{26}$ 


\section{C5. Unspecified functional bowel disorder}

An unspecified functional bowel disorder is defined as functional bowel symptoms that do not meet criteria for the previously defined categories.

\section{Functional abdominal pain}

Functional abdominal pain describes continuous, nearly continuous, or frequently recurrent pain localized in the abdomen but poorly related to gut function.

Functional abdominal pain is divided into two categories.

\section{D1. Functional abdominal pain syndrome}

Functional abdominal pain syndrome (FAPS), also called "chronic idiopathic abdominal pain" or "chronic functional abdominal pain," describes pain for at least six months that is poorly related to gut function and is associated with some loss of daily activities.

FAPS occurs in $1.7 \%$ of people, mainly women, and is associated with significant absenteeism from work and physician visits. ${ }^{5}$ Over time, patients with FAPS tend to have many specialist referrals, diagnostic tests and major abdominal and pelvic operations. ${ }^{27}$

DIAGNOSTIC CRITERIA

At least six months of:

(1) Continuous or nearly continuous abdominal pain; and

(2) No or only occasional relation of pain with physiological events (e.g., eating, defecation or menses); and

(3) Some loss of daily functioning; and

(4) The pain is not feigned (e.g., malingering); and

(5) Insufficient criteria for other functional gastrointestinal disorders that would explain the abdominal pain.

DIAGNOSTIC COMMENTS

Aspects of the patient's pain behavior are of primary importance. ${ }^{27}$ Typically, the pain is described in emotional or bizarre terms, involves a large anatomic area, is associated with other painful symptoms, and is part of a continuum of painful experiences over many years. Usually, patients urgently report pain as extremely intense, and they request many diagnostic studies or surgery, focus primarily on the illness, and relentlessly seek pain relief and validation that the pain is "organic." They often ignore or deny a role for psychosocial contributions and absolve personal responsibility for self-management, while placing high expectations for relief on the physician. Pain behavior may diminish when the patient is distracted or not aware of being observed. A spouse or parent may be so affected by the patient's illness as to report the history. Requests for narcotics are common. FAPS may co-exist with a structural disease (e.g., chronic pancreatitis) or FBD (e.g., IBS). The observation of a lack autonomic arousal, eye closure during abdominal examination, and diminished pain behavior with stethoscope application to the abdomen are typical. Multiple abdominal scars are common.

TREATMENT RECOMMENDATIONS

Management depends on an effective doctorpatient relationship, ${ }^{27}$ including reasonable goals, regular appointments and, in some cases, concurrent psychological treatment. Analgesics are ineffective, and narcotics should be avoided. Concurrent depression should be treated. Low doses of antidepressants can reduce pain as well as insomnia. Anxiolytic therapy, if used at all, should be limited in duration. Various types of psychotherapy have been tried without critical evaluation. A multidisciplinary pain management program may be the most promising approach. ${ }^{28}$

\section{D2. Unspecified functional abdominal pain}

Unspecified functional abdominal pain is functional abdominal pain which fails to meet criteria for FAPS (D1).

1 Whitehead WE, Crowell MD, Bosmajian L, et al. Existence of irritable bowel syndrome supported by factor analysis of symptoms in two community samples. Gastroenterology 1990;98:336-40.

2 Taub E, Cuevas JL, Cook EW, et al. Irritable bowel syndrome defined by factor analysis. Dig Dis Sci 1995;40: 2647-55.

3 Thompson WG. Gender differences in irritable bowel symptoms. Eur $\mathcal{f}$ Gastroenterol Hepatol 1997;9:299-302.

4 Drossman DA, Whitehead WE, Camilleri M. Irritable bowel syndrome. A technical review for practice guideline development. Gastroenterology 1997;112:2120-37.

5 Drossman DA, Li Z, Andruzzi E, et al. U.S. householder survey of functional gastrointestinal disorders: prevalence, sociodemography and health impact. Dig Dis Sci 1993;38: 1569-80.

6 Talley NJ, Gabriel SE, Harmsen WS, et al. Medical costs in community subjects with irritable bowel syndrome. Gastroenterology 1995;109:1736-41.

7 Manning AP, Thompson WG, Heaton KW, et al. Towards positive diagnosis of the irritable bowel syndrome. BMF 1978;ii:653-54.

8 Longstreth GF. Irritable bowel syndrome. Diagnosis in the managed care era. Dig Dis Sci 1997;42:1105-11.

9 Harvey RF, Mauad EC, Brown AM. Prognosis in the irritable bowel syndrome. A 5-year prospective study. Lancet 1987;i:963-5.

10 Chaudhary NA, Truelove SC. The irritable colon syndrome. $Q \mathcal{F}$ Med 1962;31:307-22.

11 Longstreth GF. Irritable bowel syndrome and chronic pelvic pain. Obstet Gynecol Surv 1994;49:505-7.

12 O'Donnell LJD, Virjee J, Heaton KW. Detection of pseudodiarrhea by simple clinical assessment of intestinal transit rate. BMF 1990;300:439-40.

13 Kruis W, Thieme $\mathrm{CH}$, Weinzieri $\mathrm{M}$, et al. A diagnostic score for the irritable bowel syndrome. Its value in the exclusion for the irritable bowel syndrome. Its value in the

14 Camilleri M, Prather CM. The irritable bowel syndrome. A review and a practical approach to management. Ann Intern Med 1992;116:1001-8.

15 Johnsen R, Jacobsen BK, Forde OH. Association between symptoms of irritable colon and psychological and social conditions and lifestyle. BMf 1986;292:1633-5.

16 Kay L, Jorgensen T, Jensen KH. The epidemiology of irritable bowel syndrome in a random population. Prevalence, incidence, natural history and risk factors. $\mathcal{F}$ Intern Med 1994;236:23-30.

17 Suarez FL, Savaiano DA, Levitt MD. A comparison of symptoms after the consumption of milk or lactosehydrolyzed milk by people with self-reported severe lactose intolerance. N Engl f Med 1995;333:1-4.

18 Everhart JE, Go VL, Johannes RS, et al. A longitudinal survey of self-reported bowel habits in the United States. Dig vey of self-reported bowel
Dis Sci 1989;34:1153-62.

19 Sandler RS, Drossman DA. Bowel habits in young adults not seeking health care. Dig Dis Sci 1987;32:841-5. 
20 Probert CSJ, Emmett PM, Cripps HA, et al. Evidence for the ambiguity of the $\mathrm{PM}$, Cripps HA, et al. Evidence for the ambiguity of the term constipation:
bowel syndrome. Gut 1994;35:1455-8.

21 van der Sijp JRM, Kamm MA, Nightingale JMD, et al. Radioisotope determination of regional colonic transit in severe constipation. Comparison with radiopaque markers. Gut 1993;34:402-8.

22 Wald A, Camara BJ, Freimanis MG, et al. Contribution of evacuation proctography and anorectal manometry to the evaluation of adults with constipation and defecatory difficulty. Dig Dis Sci 1990;25:481-7.

23 Voderholzer WA, Schatke W, Muhldorfer BE, et al. Clinical response to dietary fiber treatment of chronic constipation. Am $\mathcal{F}$ Gastroenterol 1997;92:95-8.
24 Klauser AG, Muhldorfer BE, Voderholzer WA, et al. Polyethylene glycol 4000 for slow transit constipation. $Z$ Polyethylene glycol 4000

25 Heaton KW, Radavan J, Cripps $\mathrm{H}$, et al. Defecation frequency and timing, and stool form in the general population: a prospective study. Gut 1992;33:818-24.

26 Afzalpurkar RG, Schiller LR, et al. The self-limited nature of chronic idiopathic diarrhea. N Engl F Med 1992;327:1849 52.

27 Drossman DA. Chronic functional abdominal pain. Am $\mathcal{F}$ Gastroenterol 1996;91:2270-81.

28 Kames LD, Rapkin AJ, Naliboff BD, et al. Effectiveness of an interdisciplinary pain management program for the treatment of chronic pelvic pain. Pain 1990;41:41-6.

For further information and updates on Rome II, visit our website at: www.romecriteria.org 